Invited Paper presented at 3rd International Symposium on

TRI-PP-00-18

Symmetries in Subatomic Physics, Adelaide, March 13-17

April 2000

\title{
Constraints on a Parity-even/Time-Reversal-odd Interaction ${ }^{1}$
}

\author{
Willem T.H. van Oers \\ Department of Physics, University of Manitoba, Winnipeg, MB, Canada R3T 2N2 \\ and \\ TRIUMF, 4004 Wesbrook Mall, Vancouver, BC Canada V6T $2 A 3$
}

\begin{abstract}
Time-Reversal-Invariance non-conservation has for the first time been unequivocally demonstrated in a direct measurement, one of the results of the CPLEAR experiment. What is the situation then with regard to time-reversal-invariance nonconservation in systems other than the neutral kaon system? Two classes of tests of time-reversal-invariance need to be distinguished: the first one deals with parity violating (P-odd)/time-reversal-invariance non-conserving (T-odd) interactions, while the second one deals with P-even/T-odd interactions (assuming CPT conservation this implies C-conjugation non-conservation). Limits on a P-odd/T-odd interaction follow from measurements of the electric dipole moment of the neutron. This in turn provides a limit on a $\mathrm{P}$-odd/T-odd pion-nucleon coupling constant which is $10^{-4}$ times the weak interaction strength. Limits on a P-even/T-odd interaction are much less stringent. The better constraint stems also from the measurement of the electric dipole moment of the neutron. Of all the other tests, measurements of charge-symmetry breaking in neutron-proton elastic scattering provide the next better constraint. The latter experiments were performed at TRIUMF (at 477 and $347 \mathrm{MeV}$ ) and at IUCF (at $183 \mathrm{MeV}$ ). Weak decay experiments (the transverse polarization of the muon in $K^{+} \rightarrow \pi^{0} \mu^{+} \nu_{\mu}$ and the transverse polarization of the positrons in polarized muon decay) have the potential to provide comparable or possibly better constraints.
\end{abstract}

\section{INTRODUCTION}

Time-reversal-invariance non-conservation has for the first time been unequivocally demonstrated in a direct measurement in the CPLEAR experiment. [1] The experiment measured the difference in the transition probabilities $P\left(\bar{K}^{0} \rightarrow K^{0}\right)$ and $P\left(K^{0} \rightarrow \bar{K}^{0}\right)$. Assuming CPT conservation but allowing for a possible breaking of the $\Delta \mathrm{S}=\Delta \mathrm{Q}$ rule, the result obtained for $A_{T}$

1) Work supported in part by the Natural Sciences and Engineering Research Council of Canada. 


$$
\left.A_{T}=\frac{R\left(\bar{K}^{0} \rightarrow K^{0}\right)-R\left(K^{0} \rightarrow \bar{K}^{0}\right)}{R\left(\bar{K}^{0} \rightarrow K^{0}\right)+R\left(K^{0} \rightarrow \bar{K}^{0}\right)}=[6.6 \pm 1.3 \text { (stat.) } \pm 1.0 \text { (syst. })\right] \times 10^{-3}
$$

is in good agreement with the measure of $\mathrm{CP}$ violation in neutral kaon decay. A more recent reported result is a large asymmetry in the distribution of $K_{L} \rightarrow$ $\pi^{+} \pi^{-} e^{+} e^{-}$events in the CP-odd/T-odd angle $\phi$ between the decay planes of the $\pi^{+} \pi^{-}$and $e^{+} e^{-}$pairs in the $K_{L}$ centre of mass system. The overall asymmetry found was $[13.6 \pm 2.5$ (stat.) \pm 1.2 (syst.) $] \%$. [2] The question then is: what is the situation with regard to time-reversal-invariance in systems other than the kaon system?

Tests of time-reversal-invariance can be distinguished as belonging to two classes: the first one deals with $\mathrm{P}$-odd/T-odd interactions, while the second one deals with $\mathrm{P}$-even/T-odd interactions (assuming CPT conservation this implies C-conjugation non-conservation). But it should be noted that constraints on these two classes of interactions are not independent since the effects due to P-odd/T-odd interactions may also be produced by $\mathrm{P}$-even/T-odd interactions in conjunction with Standard Model parity violating radiative corrections. The latter can occur at the $10^{-7}$ level and consequently could present a limit on the constraint of a P-even/T-odd interaction, derived from experiment. Limits on a P-odd/T-odd interaction follow from measurements of the electric dipole moment of the neutron (which currently stands at $<6 \times 10^{-26}$ e.cm [95\% C.L.]). This provides a limit on a P-odd/T-odd pion-nucleon coupling constant which is less than $10^{-4}$ times the weak interaction strength. Measurements of ${ }^{129} \mathrm{Xe}$ and ${ }^{199} \mathrm{Hg}\left(<8 \times 10^{-28}\right.$ e.cm [95\% C.L.] $)$ give similar constraints. [see Ref. 3]

Experimental limits on a P-even/T-odd interaction are much less stringent. Following the standard approach of describing the nucleon-nucleon interaction in terms of meson exchanges, it can be shown that only charged rho-meson exchange and $\mathrm{A}_{1^{-}}$ meson exchange can lead to a P-even/T-odd interaction. [4] The better constraints stem from measurements of the electric dipole moment of the neutron and next from measurements of charge symmetry breaking in neutron-proton $(n-p)$ elastic scattering. All other experiments, like gamma decay experiments [5], detailed balance experiments [6], polarization - analyzing power difference measurements [7], and five-fold correlation experiments with polarized incident nucleons and aligned nuclear targets [8], have been shown to be at least an order of magnitude less sensitive. Haxton, Hoering, and Musolf [3] have deduced constraints on a P-even/T-odd interaction from nucleon, nuclear, and atomic electric dipole moments with the better constraint coming from the electric dipole moment of the neutron. In terms of a ratio to the strong rho-meson nucleon coupling constant, they deduced for the P-even/T-odd rho-meson nucleon coupling: $\left|\overline{g_{\rho}}\right|<0.53 \times 10^{-3} \times\left|f_{\pi}^{\mathrm{DDH}} / f_{\pi}^{\text {meas. }}\right|$. But the ratio of the theoretical to the experimental value of $f_{\pi}$ may be as large as 15 ! [9] However, constraints derived from one-loop contributions to the electric dipole moment of the neutron exceed the two-loop limits by more than an order of magnitude and are much more stringent. [10] However, a translation in terms of coupling strengths in the hadronic sector still needs to be made. 
It is very difficult to accommodate a P-even/T-odd interaction in the Standard Model. It requires $\mathrm{C}$-conjugation non-conservation, which cannot be introduced at the first generation quark level. It can neither be introduced into the gluon selfinteraction. Consequently, one needs to consider C-conjugation non-conservation between quarks of different generations and/or between interacting fields. [11]

\section{CHARGE SYMMETRY BREAKING IN NEUTRON PROTON ELASTIC SCATTERING}

Charge symmetry breaking (CSB) in neutron-proton elastic scattering manifests itself as a non-zero difference of the neutron $\left(A_{n}\right)$ and proton $\left(A_{p}\right)$ analyzing powers, $\Delta A=A_{n}-A_{p}=2 \times\left[\operatorname{Re}\left(b^{*} f\right)+\operatorname{Im}\left(c^{*} h\right)\right] / \sigma_{0}$. Here the complex amplitude $f$ is charge symmetry breaking, while the complex amplitude $h$ is both charge symmetry breaking and time-reversal-invariance non-conserving. The complex amplitudes $b$ and $c$ belong to the usual five $n$ - $p$ scattering amplitudes and $\sigma_{0}$ is the unpolarized differential cross section. The three precision experiments performed (at TRIUMF at $477 \mathrm{MeV}$ [12] and at $347 \mathrm{MeV}$ [13], and at IUCF at $183 \mathrm{MeV}$ [14]) have unambiguously shown that charge symmetry is broken and that the results for $\Delta A$ at the zero-crossing angle of the average analyzing power are very well reproduced by meson exchange model calculations (see Fig. 1). A P-even/T-odd interaction produces a term in the scattering amplitude which is simultaneously charge symmetry breaking (the complex amplitude $h$ in the expression above). Thus, Simonius [15] deduced an upper limit on a P-even/T-odd CSB interaction from a comparison of the experimental results with the theoretical predictions for the three $n-p$ CSB experiments. The upper limit so derived is $\left|\bar{g}_{\rho}\right|<6.7 \times 10^{-3}$ [95\% C.L.]. This is therefore comparable to the upper limit deduced from the electric dipole moment of the neutron, taking present experimental limits of $f_{\pi}$, and is considerably lower than the limits inferred from direct tests of a P-even/T-odd interaction. For instance the detailed balance experiments give a limit of $\left|\bar{g}_{\rho}\right|<2.5 \times 10^{-1}$ [see Ref. 8], while measurements of the five-fold correlation parameter $A_{y, x z}$ in polarized neutron transmission through nuclear spin-aligned ${ }^{165}$ Ho give a limit of $\left|\bar{g}_{\rho}\right|<5.9 \times 10^{-2}$ even though the measured value of $A_{y, x z}$ was $(8.6 \pm 7.7) \times 10^{-6}$. [8] It is effectively only the valence proton in ${ }^{165}$ Ho which contributes to $A_{y, x z}$. Even though it is inconceivable in the Standard Model to account for a P-even/T-odd interaction, there is a need to clarify the experimental situation by providing a better experimental result.

Such an experimental constraint may be provided by an improved upper limit on the electric dipole moment of the neutron. In fact a new measurement with a sensitivity of $4 \times 10^{-28}$ e.cm has been proposed at the Los Alamos Neutron Science Center. [16] Performing an improved $n$ - $p$ elastic scattering CSB experiment also appears to be a very attractive possibility. One can calculate with a great deal of confidence the contributions to CSB due to one-photon exchange and due to the $n$ -

$p$ mass difference affecting charge one-pion and rho-meson exchange. Furthermore, 


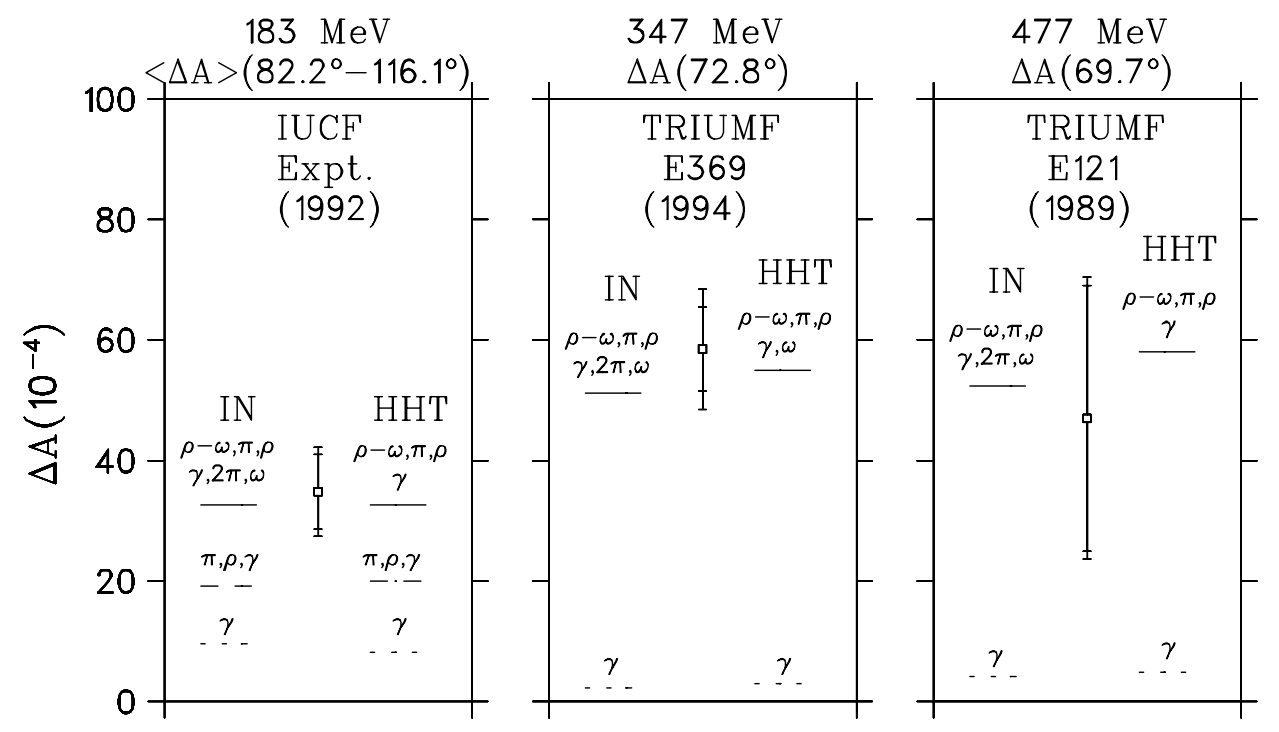

FIGURE 1. Experimental results of $\Delta A$ at the zero-crossing angle at incident neutron energies of 183, 347, and $477 \mathrm{MeV}$ compared with theoretical predictions of Iqbal and Niskanen, and Holzenkamp, Holinde, and Thomas. The inner error bars present the statistical uncertainties; the outer error bars have the systematic uncertainties included (added in quadrature). For further details see Ref. 13 .

one can select an energy where the $\rho^{\circ}-\omega$ meson mixing contribution changes sign at the same angle where the average of the analyzing powers $A_{n}$ and $A_{p}$ changes sign and therefore does not contribute to $\Delta A$. This occurs at a neutron energy of $320 \mathrm{MeV}$ and is caused by the particular interplay of the $n-p$ phase shifts and the form of the spin/isospin operator connected with the $\rho^{\circ}-\omega$ mixing term. But also the one-photon exchange term changes sign at about the same angle at $320 \mathrm{MeV}$. The contribution due to two-pion exchange with an intermediate $\Delta$ is expected to be less than one tenth of the overall CSB effect, essentially determining an upper limit on the theoretical uncertainty (see Fig. 2). [17] It has been shown that simultaneous $\gamma-\pi$ exchanges can only contribute to $\Delta A$ through second order processes and can therefore be neglected. [18] Also the effects of inelasticity are negligibly small at $320 \mathrm{MeV}$. It appears therefore well within reach to reduce the theoretical uncertainty in the comparison of experiment and theory. Subtracting the calculated $\Delta A$ from the measured $\Delta A$ permits establishing an upper limit on a P-even/T-odd/CSB interaction.

In the TRIUMF CSB experiments polarized neutrons were scattered from unpolarized protons and vice versa. The polarized (or unpolarized) neutron beam was obtained using the $(p, n)$ reaction with a 369 (and 497 ) $\mathrm{MeV}$ polarized (or unpolarized) proton beam incident on a $0.20 \mathrm{~m}$ long $\mathrm{LD}_{2}$ target. At these energies one makes use of the large sideways-to-sideways polarization transfer coefficient $r_{t}$ at $9^{\circ}$ in the lab. The only difference in obtaining the polarized and unpolarized $347 \mathrm{MeV}$ neutron beams was turning off the pumping laser light in the optically pumped polarized ion source (OPPIS). The polarized proton target was of the frozen spin type 

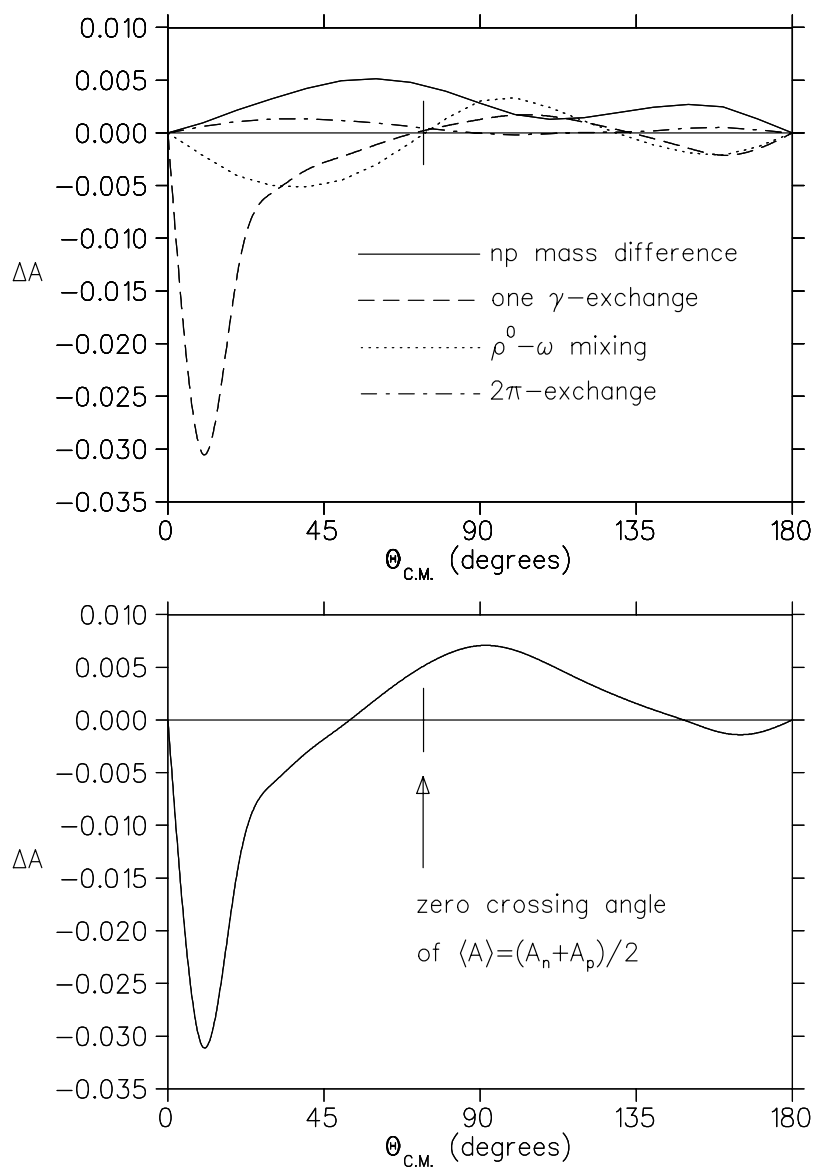

FIGURE 2. Angular distributions of the different contributions to $\Delta A$ at an incident neutron energy of $320 \mathrm{MeV}$. (Ref. 17) Note that the $\rho^{0}-\omega$ mixing contribution passes through zero at the same angle as the average of $A_{n}$ and $A_{p}$ (vertical bar). The lower part of the figure gives the total $\Delta A$ angular distribution.

with butanol beads as target material. The same target after depolarization was used as the unpolarized proton target. Great care was taken that the two interleaved phases of the experiments were performed with identical beam and target parameters except for the polarization states. At $347 \mathrm{MeV}$ scattered neutrons and recoiling protons were detected in coincidence in the c.m. angular range 53.4 to 86.9 degrees in two left-right symmetric detector systems. Rather than measuring $A_{n}$ and $A_{p}$ directly (which would limit the accuracy attainable by not having polarization calibration standards of the required precision), the zero-crossings of $A_{n}$ and $A_{p}$ were determined by fitting the partial angular distributions with polynomials, deduced/. from $n-p$ phase shift analyses. The difference $\Delta A$ followed then by multiplying the difference in the zero-crossing angles by the average slope of the analyzing powers (the experiment measured the slope of $A_{p}$ at the zero-crossing angle, which is a good approximation for the average slope at the zero-crossing angle and introduces a negligible error). The execution of the experiments depended 
on a great deal of simultaneous monitoring and online control measurements. Both the statistical and systematic errors, obtained in the $347 \mathrm{MeV}$ experiment, can be considerably improved upon (by a factor three to four). With the OPPIS developments which have taken place in the intervening years and with a biased Na-ionizer cell it will be possible to obtain up to $50 \mu \mathrm{A}$ of $342 \mathrm{MeV} 80 \%$ polarized proton beam incident on the neutron production target (a factor of 50 increase in neutron beam intensity at $320 \mathrm{MeV}$ over the previous $347 \mathrm{MeV}$ CSB experiment). In addition various systematic error reducing improvements can be introduced in the experimental arrangements and procedures. Such an experiment would constitute a measurement of CSB in $n$ - $p$ elastic scattering of unprecedented precision of great value on its own and would simultaneously provide a greatly improved upper limit on a P-even/T-odd interaction.

\section{PARTICLE DECAYS}

Searches for P-even/T-odd interactions are also made in particle decays, e.g., in the decay $\mu^{+} \rightarrow e^{+} \nu_{e} \bar{\nu}_{\mu}$ and in the decay $K^{+} \rightarrow \mu^{+} \pi^{0} \nu_{\mu}$. A non-zero value of the muon polarization transverse to the decay plane would be an indication of timereversal-invariance non-conservation. An experiment to measure the first decay is presently being executed at PSI. [19] Several experiments have been performed to measure the transverse muon polarization in both neutral and charged kaon decay. There is a unique feature to the transverse muon polarization in that it does not have contributions from the Standard Model at tree level and that higher order effects are of order $10^{-6}$. When only one charged particle is present in the final state, a final state interaction, which can mimic a time-reversal-invariance breaking effect, is greatly reduced and is estimated to occur only at the same level of $10^{-6}$. The more recent effort of measuring the time-reversal-invariance non-conserving transverse muon polarization is being done at KEK using a stopped $K^{+}$beam. The experiment reported a result for $\mathrm{P}_{T}=-0.0042 \pm 0.0049$ (stat.) \pm 0.0009 (syst.), based on the data taken in 1996 and 1997, which translates into a value of $\operatorname{Im} \xi=$ $-0.013 \pm 0.016$ (stat.) \pm 0.003 (syst.). [20] The quantity $\xi$ is defined as the ratio of two form factors, $f_{+}\left(q^{2}\right)$ and $f_{-}\left(q^{2}\right)$, in the $K_{\mu 3}$ decay. $\operatorname{Im} \xi$ must be equal to zero for time-reversal-invariance to hold. With the data already in hand and with the approved data taking time, it is anticipated to arrive at a statistical error of \pm 0.008 in $\operatorname{Im} \xi$. The best previous experimental limits were obtained with both neutral and charged kaons at the BNL-AGS. [21] A combination of both experimental results provided a limit on the imaginary part of the hadron form factor, $\operatorname{Im} \xi=-0.010$ \pm 0.019 . A new search for the time-reversal-invariance non-conserving transverse muon polarization with in-flight decays of $K^{+} \rightarrow \mu^{+} \pi^{0} \nu_{\mu}$ was proposed at the BNL-AGS. [22] It was intended to obtain a sensitivity to the transverse muon polarization of \pm 0.00013 , corresponding to a sensitivity to $\operatorname{Im} \xi$ of \pm 0.0007 . Similar searches for the time-reversal-invariance non-conserving transverse $\tau$ polarization in B semileptonic decays, $B \rightarrow M \tau \nu_{\tau}$ are under consideration. Significant transverse $\tau$ 
lepton polarizations have been predicted. Clearly, a non-zero value of the transverse muon polarization in $K_{\mu 3}$ decay, or of the $\tau$ lepton in semi-leptonic $B$ decays would constitute evidence for new physics.

\section{SUMMARY}

The searches made so far for a P-even/T-odd interaction have resulted in only very modest constraints on such an interaction. Most promising are the continued efforts to measure the electric dipole moment of the neutron and secondly charge symmetry breaking in neutron-proton elastic scattering at around $320 \mathrm{MeV}$. But also measurements of transverse lepton polarizations in $\mu, K$, and $B$ decays have the potential to set better experimental limits on a P-even/T-odd interaction.

\section{REFERENCES}

1. Angelopoulos, A. et al., (CPLEAR Collaboration), Phys. Lett. B444, 43 (1998).

2. Alavi-Harati, A. et al., (KTeV Collaboration), Phys. Rev. Lett. 84, 408 (2000).

3. Haxton, W.C., Hoering, A. and Musolf, M.J., Phys. Rev. D50, 3422 (1994).

4. Simonius, M., Phys. Lett. 58B, 147 (1975).

5. Boehm, F. in Symmetries and Fundamental Interactions in Nuclei, ed. Haxton, W.C. and Henley, E.M. (World Scientific, Singapore, 1997), p. 67.

6. Blanke, E. et al., Phys. Rev. Lett. 51, 355 (1983).

7. Conzett, H.E. in Polarization Phenomena in Nuclear Physics - 1980, ed. Ohlsen, G.G., Brown, R.E., Jarmie, N., McNaughton, W.W., and Hale, G.M. AIP Conference Proceedings No 69, p. 1452.

8. Huffman, P.R. et al., Phys. Rev. C55, 2684 (1997).

9. Page, S.A. et al., Phys. Rev. C35, 1119 (1987); Bini, M. et al., Phys. Rev. C38, 1195 (1988).

10. Ramsey-Musolf, M.J., Phys. Rev. Lett. 83, 3997 (1999).

11. Simonius, M. in Intersections between Particle and Nuclear Physics - 1984, ed. Mischke, R.E. AIP Conference Proceedings No 123, p. 1115.

12. Abegg, R. et al., Phys. Rev. D39, 2464 (1989).

13. Zhao, J. et al., Phys. Rev. C57, 2126 (1998).

14. Vigdor, S.E. et al., Phys. Rev. C46, 410 (1992).

15. Simonius, M. Phys. Rev. Lett. 78, 4161 (1997).

16. LANSCE Proposal, spokespersons Cooper, M.J. and Lamoreaux, S.K.

17. Iqbal, J. private communication (1999).

18. Friar, J.L. and Coon, S.A. Phys. Rev. C53, 588 (1996).

19. Fetscher, W. these Proceedings.

20. Abe, M. et al., Phys. Rev. Lett. 83, 4253 (1999).

21. Morse, W.M. et al., Phys. Rev. D21, 1750 (1980); Blatt, S.R. et al., Phys. Rev. D27, 1056 (1983).

22. BNL-AGS proposal, spokespersons Divan, M.V., Ma, Hong and Adair, R. 